framum

Sociológico

\section{Forum Sociológico}

Série II

$27 \mid 2015$

Mobilidade científica \& imigração qualificada

\title{
Movilidad de científicos franceses a México
}

Movilidad de científicos franceses a México ${ }^{1}$

Sylvie Didou Aupetit e María Cecilia Oviedo Mendiola

\section{(2) OpenEdition}

Journals

Edição electrónica

URL: https://journals.openedition.org/sociologico/1324

DOI: $10.4000 /$ sociologico.1324

ISSN: 2182-7427

\section{Editora}

CICS.NOVA - Centro Interdisciplinar de Ciências Sociais da Universidade Nova de Lisboa

Edição impressa

Data de publição: 21 dezembro 2015

Paginação: 23-30

ISSN: 0872-8380

\section{Refêrencia eletrónica}

Sylvie Didou Aupetit e María Cecilia Oviedo Mendiola, «Movilidad de científicos franceses a México», Forum Sociológico [Online], 27 | 2015, posto online no dia 28 maio 2016, consultado o 31 março 2022. URL: http://journals.openedition.org/sociologico/1324 ; DOI: https://doi.org/10.4000/sociologico.1324 


\title{
MOVILIDAD DE CIENTÍFICOS FRANCESES A MÉXICO ${ }^{1}$
}

\author{
Sylvie Didou Aupetit \\ Centro de Investigación y Estudios Avanzados, Departamento de Investigaciones Educativas (Cinvestav-DIE) \\ María Cecilia Oviedo Mendiola \\ Centro de Investigación y Estudios Avanzados, Departamento de Investigaciones Educativas (Cinvestav-DIE)
}

\begin{abstract}
Resumo
Analisamos os perfis da migração de cientistas estrangeiros contratados de forma fixa pelas instituições de educação superior mexicana, suas condições de profissionalização e suas redes, como ferramentas para situar-se em um âmbito de investigação científica, ao mesmo tempo altamente internacionalizado e profundamente arraigado em contextos locais e institucionais. As hipóteses centrais são: a) A migração influencia de forma duradoura na trajetória dos pesquisadores; b) A escala sistêmica, no México, é uma estratégia de consolidação das capacidades instaladas da indagação e pós-graduação; c) É uma aposta dos grupos científicos para melhorar alguns indicadores de qualidade e suportar os problemas acarreados pelo baixo nível de rotação e o envelhecimento da corpo científico.
\end{abstract}

Palavras-chave: migração científica; mobilidade internacional; redes

\section{Abstract}

We analyze the profiles of foreign scientists granted with definitive posts by Mexican higher education institutions, the conditions relating to their professionalization and their networks as tools for situating themselves in a scientific research context that is both highly internationalized and profoundly rooted in local and institutional contexts. The central hypotheses are: a) Migration has a lasting influence on researchers' career paths; b) On a system-wide scale, in Mexico, it is a strategy for consolidation of installed research capacities and, c) It is a bid by scientific groups to improve some quality indicators and overcome the problems entailed in the low level of turnover and ageing science staff.

Keywords: scientific migration; international mobility; networks

\section{Resumen}

Analizamos los perfiles de migración de los científicos extranjeros contratados en forma definitiva por instituciones de educación superior mexicanas, sus condiciones de profesionalización y sus redes, en tanto herramientas para situarse en un ámbito de investigación científica, a la par altamente internacionalizado y profundamente arraigado en contextos locales e institucionales. Las hipótesis centrales son: a) La migración influye durablemente la trayectoria de los investigadores; b) A escala sistémica, en México, es una estrategia de consolidación de las capacidades instaladas de indagación y posgrado; c) Es una apuesta de los grupos científicos para mejorar algunos indicadores de calidad y sobrellevar los problemas acarreados por el bajo nivel de rotación y el envejecimiento de la plantilla científica.

Palabras claves: migración científica; movilidad internacional; redes 
La movilidad científica internacional es un tema cuya actualidad se ha incrementado (Teichler, 2015). Los especialistas abordan sus componentes estructurales (mecanismos, programas, contextos) y analizan las características, situaciones profesionales, condiciones de migración y especializaciones de los científicos extranjeros. A su vez, la visibilidad de las líneas de investigación que les conciernen es acentuada esencialmente en los lugares considerados, con o sin razón, como sus principales polos de atracción: en Estados Unidos, los especialistas establecieron de qué países proceden (Appelt et al, 2015), en qué instituciones y áreas disciplinarias se graduaron (Espenshade et al., 2001), cuáles puestos y funciones desempeñan (Sabharwal, 2008), cuáles son sus grados de satisfacción en relación a sus empleos (Lin et al., 2009) o su productividad y pautas de publicación (Franzoni et al., 2013; Leydesdorff et al, 2013). En una perspectiva de comparación, la OCDE encargó estudios multinacionales sobre la circulación de talentos pero esos involucraron poco América Latina (Brasil en Franzoni et al, 2012).

Este artículo se centra en las condiciones de inserción en el mercado académico y en las redes de los científicos franceses, sean disciplinarias, estratégicas o afectivas. En México, si bien se conocen algunos de sus rasgos sociodemográficos, su participación en actividades de colaboración internacional está insuficientemente documentada. Por ello, revisamos la literatura y presentamos algunos datos sobre la composición del sub-grupo, recurriendo principalmente a la información del Sistema Nacional de Investigadores (SNI): el S.N.I selecciona a los científicos más productivos del país y agrupa aproximadamente el $20 \%$ de la plantilla (Galaz et al., 2009). Reflexionamos sobre sus características en relación a las de los científicos extranjeros en conjunto y sus rasgos de identidad. Analizamos 21 entrevistas relativas a sus relaciones profesionales con Francia versus las que mantienen en México y otros países, para caracterizar mejor sus procesos de "internacionalización".

\section{Científicos franceses en México: un tema emergente para la investigación educativa}

En América Latina, la situación de los científicos extranjeros hoy día y el brain gain asociado a su presencia son temas poco atendidos. El brain drain concentra en efecto tradicionalmente la atención de los expertos. Los estudios encontrados, esencialmente de corte histórico, vierten sobre los aportes de "celebridades" a la consolidación de corrientes de pensamiento. En fechas recientes, no obstante, algunas publicaciones han evaluado los programas de atracción de científicos extranjeros (Castaños et al., 2004; Castaños, 2009), sus contribuciones disciplinarias (Durand, 2013) o sus características socio-demográficas y profesionales (Oviedo, 2015).
Los intercambios de ideas y técnicas entre Francia y México se han tejido desde el siglo XVIII mediante expediciones científicas, órganos de prensa y exportación de instrumentos. En el XIX, entre 1820 y 1830,130 de los 1800 migrantes franceses a México eran profesionales (Meyer, $s, f,: 13$ ). En el $\mathrm{XX}$, el gobierno mexicano otorgó el asilo a intelectuales franceses durante la segunda Guerra Mundial (Rolland, 2002), hecho que explicó la creación en 1944 del Instituto Francés de América Latina (IFAL), una pieza maestra en el dispositivo de cooperación cultural y académica de Francia en México (Bataillon y Giraud, 1986). Esas influencias reciprocas nutrieron una "sensibilidad común" de larga data (Pérez Siller y Cramaussel, 2004).

Sin embargo, se sabe poco de la movilidad científica de Francia hacia México, hoy día. Los estudios más numerosos son biográficos: homenajean los científicos distinguidos que han (re)fundado ciertas disciplinas (en geografía urbana - Stamm, 2009; en historia - Hebrard, 2012). Los recopilados sobre el brain gain conciernen sobre todo el retorno de los investigadores mexicanos formados en el extranjero (Gérard, 2013) en disciplinas particulares (Grediaga y Maldonado, 2014), la constitución en universidades mexicanas de "escuelas de pensamiento" animadas por profesores formados en Francia (Agulhon, 2015) o el papel desempeñado por las grandes escuelas francesas en la formación de las elites económicas y empresariales (Wagner y García, 2015). Analizan los andamiajes de la cooperación bilateral (Didou, 2014a), sus alcances institucionales (Villavicencio, 2014) y el papel de las agrupaciones que la puentean (Huerta, 2006). Averiguan cuáles conocimientos circulan entre Francia y México, en ambas direcciones, analizando los contenidos de revistas especializadas (Bertrand y Pailler, 2013; Heymann, 2015; Albuquerque, 2015) o las co-autorias (Ainsworth, 2015).

Aunque la dispersión de los indicadores y su naturaleza heterogénea impiden tener una visión coherente de logros y problemas, el Portal Franco- Mexicano de Investigación Científica, abierto por la Embajada de Francia en México, el CONACYT y otras agencias de cooperación, en marzo $2015^{2}$, registra que unos 500 investigadores intervienen en ella y producen unas 600 co-publicaciones por año ${ }^{3}$. Esa colaboración descansa en parte en las actividades del grupo focal objeto de ese artículo.

Los franceses en el Sistema Nacional de Investigadores (S.N.I)

Como lo mencionamos antes, escasean las investigaciones referidas a los investigadores nacidos en Francia y radicados en México, independientemente de si se nacionalizaron mexicanos y del lugar donde se titularon (Didou, 2014b), probablemente debido al tránsito entre una época gloriosa a una 
caracterizada, según la expresión utilizada por Walter y Scheltzer (2014), por una "rutinización del carisma".

De acuerdo con la base de datos del S.N.I, en 2013, 2,358 investigadores de los 19,632 vigentes nacieron fuera de México. Entre 2009 y 2013, su tasa interna de aumento fue de $8.8 \%$ pero su participación relativa en el conjunto de los investigadores SNI disminuyó de 14 a 12\% (con base para 2009 en Didou y Durand, 2013: 71). El porcentaje de franceses en cambio creció en $15 \%$, pasando de 98 a 113 , el de los colombianos en $37 \%$ (al pasar de 107 a 147) y el de los cubanos en $19 \%$ (de 156 a 187) (Didou y Durand, 2013).

Entre los stocks de investigadores extranjeros en el SNI, destacan los compuestos por quienes nacieron en España y Estados Unidos, seguidos por los de Argentina, Cuba, Rusia, Colombia, Francia y Alemania: sumadas, esas poblaciones constituyen más de la mitad de los investigadores extranjeros en el SNI (ver cuadro 1).

Cuadro $1 \triangleright$ Investigadores extranjeros en el SNI

\section{3}

\begin{tabular}{lc}
\multicolumn{1}{c}{ País de nacimiento } & No. \\
\hline España & 262 \\
\hline Estados Unidos & 201 \\
\hline Argentina & 191 \\
\hline Cuba & 187 \\
\hline Colombia & 147 \\
\hline Rusia & 159 \\
\hline Francia & 113 \\
\hline Alemania & 106 \\
\hline Subtotal & 1,353 \\
\% & $57 \%$ \\
\hline Otros & 1,005 \\
\% & $43 \%$ \\
\hline Total & 2,358 \\
\% & $100 \%$ \\
\hline$---\cdot-\cdot-\cdot-\cdot$. \\
\hline
\end{tabular}

Fuente: CONACYT, SNI Investigadores vigentes, agosto 2013. Fuente: CONACYT, SNI Investigadores vigentes, agosto 2013.

Considerados como grupo, los investigadores franceses tienen algunas características que los distinguen del conjunto de la población del SNI: 1) un elevado número de mujeres; 2 ) una presencia en los niveles II y III; 3) una membresía más numerosa en las áreas IV y $\mathrm{V}$.

Aunque con una composición mayoritariamente masculina, el $42 \%$ del grupo está compuesto por mujeres, lo que es una alta proporción si se compara ese dato con el relativo al conjunto de los miembros del SNI (34\%). Esa mayor presencia femenina es igual a la constatada entre los investigadores nacidos en España; sólo es superada por el número de investigadoras nacidas en Argentina (89), que representan el $47 \%$ de quienes nacieron en ese país.

La mayoría de los investigadores franceses en el SNI realizó sus estudios de doctorado en su país natal - 78 investigadores, lo que equivale al $69 \%$ del total -; $16-14 \%$ - hizo su doctorado en México y el resto en un país tercero. Las entrevistas indicaron dos pautas predominantes, la obtención de un doctorado antes de migrar a México o un retorno al país de origen con ese motivo después de fijar la residencia en México. En este último caso, el interés es intelectual pero también afectivo:

Decido ir a hacer el doctorado en Francia. Coincide que, en ese momento, viene $* * *$, da un seminario y yo creo que tengo la idea de ir. Una idea ambigua, que es de formación pero también de deseo de ir a Francia recuperar un poco este pasado y todo esto. (Entrevistada n. 1)

Respecto del nivel al que pertenecen, $41 \%$ de los investigadores franceses se ubican en los niveles II y III, mientras que el porcentaje para el conjunto de los miembros del Sistema es de $27 \%$. Esta concentración en los niveles II y III del Sistema es común a casi todos los grupos de investigadores extranjeros, con excepción de los cubanos y colombianos (ver gráfico 1 ). Entre los nacidos en México, sólo el $24 \%$ pertenece a los niveles II y III.

Algunos investigadores franceses señalaron la importancia de un "habito académico" adquirido en Francia que favorece y naturaliza la producción escrita además de proporcionar habilidad para planear la organización del proceso de indagación:

Aquí me dicen icuánto producesi pero, para mí, es el trabajo normal: tampoco siento que me estoy matando, sí, tengo buen ritmo pero esa es la diferencia que yo vería entre un sistema u otro. En México, a veces, hay un cierto relajamiento que perturba la continuidad o la constancia en el trabajo. Como me pidió franqueza, otro punto es el método, saber cómo conducir con cierta lógica [un proyecto], trazarse un camino en la investigación por etapas, por metas: eso nos lo han enseñado desde una edad temprana: para nosotros, es bastante natural. (Entrevistada n. 7)

Otro característica del grupo de investigadores franceses en el SNI es su distribución por áreas de conocimiento: se concentra principalmente en las áreas IV - Humanidades y Ciencias de la Conducta y 
V - Ciencias Sociales, mientras que la población total del Sistema se distribuye más homogéneamente, con una mayor presencia en las áreas II - Biología y Química y I - Físico-Matemáticas y Ciencias de la Tierra (ver cuadro 2).
De los investigadores nacidos en Francia adscritos al área IV, 9 de 15 son jóvenes, de entre 34 y 45 años. En el área V, 7 de 12 se encuentran en ese mismo rango de edades, es decir están probablemente consolidando su carrera académica.

Gráfico $1 \triangleright$ Investigadores en el SNI por país de nacimiento y nivel al que pertenecen (\%)

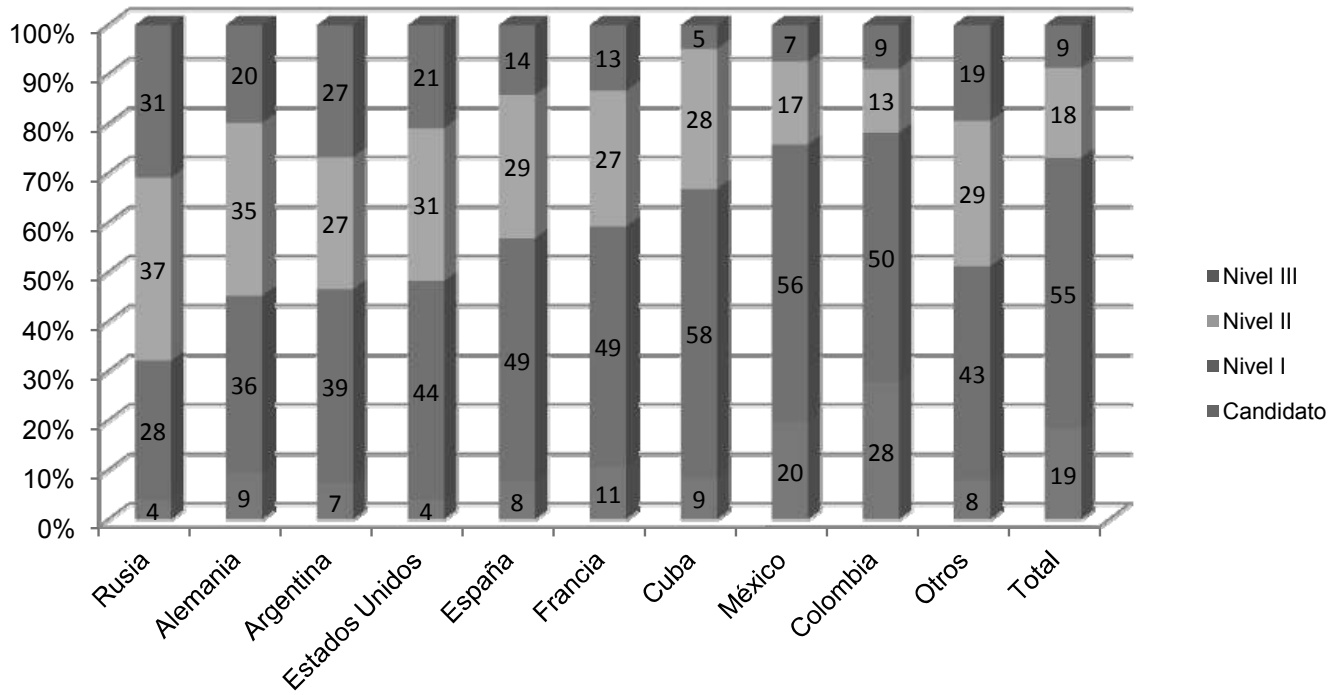

Fuente: CONACYT, SNI Investigadores vigentes, agosto 2013

Cuadro $2 \downarrow$ Investigadores nacidos en Francia y total de investigadores en el SNI por nivel y área de conocimiento

2013

\begin{tabular}{|c|c|c|c|c|c|c|c|c|c|c|}
\hline \multirow[b]{2}{*}{ Área $* /$ Nivel } & \multicolumn{5}{|c|}{ Investigadores nacidos en Francia } & \multicolumn{5}{|c|}{ Investigadores SNI Total } \\
\hline & Candidato & Nivel I & Nivel II & Nivel III & $\begin{array}{c}\text { Total } \\
\%\end{array}$ & Candidato & Nivel I & Nivel II & Nivel III & $\begin{array}{c}\text { Total } \\
\%\end{array}$ \\
\hline I & 2 & 10 & 9 & 1 & $\begin{array}{c}22 \\
19.5 \%\end{array}$ & 604 & 1,386 & 742 & 448 & $\begin{array}{c}3,180 \\
16.2 \%\end{array}$ \\
\hline II & & 8 & 2 & 3 & $\begin{array}{c}13 \\
11.5 \%\end{array}$ & 625 & 1,854 & 558 & 297 & $\begin{array}{c}3,334 \\
17.0 \%\end{array}$ \\
\hline III & & 3 & 2 & & $\begin{array}{c}5 \\
4.4 \%\end{array}$ & 262 & 1,240 & 339 & 179 & $\begin{array}{c}2,020 \\
10.3 \%\end{array}$ \\
\hline IV & 4 & 15 & 6 & 7 & $\begin{array}{c}32 \\
28.3 \%\end{array}$ & 403 & 1,636 & 615 & 254 & $\begin{array}{l}2,908 \\
14.8 \%\end{array}$ \\
\hline $\mathrm{V}$ & 3 & 12 & 9 & 4 & $\begin{array}{c}28 \\
24.8 \%\end{array}$ & 493 & 1,691 & 527 & 264 & $\begin{array}{l}2,975 \\
15.2 \%\end{array}$ \\
\hline VI & 1 & 1 & 3 & & $\begin{array}{c}5 \\
4.4 \%\end{array}$ & 520 & 1,319 & 342 & 134 & $\begin{array}{l}2,315 \\
11.8 \%\end{array}$ \\
\hline VII & 2 & 6 & & & $\begin{array}{c}8 \\
7.1 \%\end{array}$ & 727 & 1,597 & 451 & 125 & $\begin{array}{l}2,900 \\
14.8 \%\end{array}$ \\
\hline Total & 12 & 55 & 31 & 15 & $\begin{array}{c}113 \\
100.0 \%\end{array}$ & 3,634 & 10,723 & 3,574 & 1,701 & $\begin{array}{r}19,632 \\
100.0 \%\end{array}$ \\
\hline
\end{tabular}

* Área I Físico-Matemáticas y Ciencias de la Tierra; Área II Biología y Química; Área III Medicina y Ciencias de la Salud; Área IV Humanidades y Ciencias de la Conducta; Área V Ciencias Sociales; Área VI Biotecnología y Ciencias Agropecuarias; Área VII Ingenierías

Fuente: CONACYT, SNI Investigadores vigentes, agosto 2013 
La importante presencia de investigadores franceses en las áreas IV y $\mathrm{V}$ coincide con la que tienen los investigadores extranjeros en esas mismas áreas: en 2013, el área I recibía a 586 investigadores nacidos en el extranjero, que representan el $25 \%$ del total; el área IV agrupaba a 545, que representaban el $23 \%$ y el área $V$ a 438 , equivalentes al $19 \%$ (ver gráfico 2). En cambio, en las áreas IV, I y V del SNI, sólo 19,18 y 15 de cada 100 investigadores son extranjeros, respectivamente.

Ningún investigador francés registrado en el SNI en 2013 tiene menos de 33 años, lo que coincide con una pertenencia muy escasa de investigadores extranjeros menores de 30 años: sólo 1 de un total de 25 investigadores está en esa condición.

Un pie aquí, un pie allá: redes y formas asociativas

Una vez establecidos los rasgos generales de ese grupo, entrevistamos a 21 investigadores franceses (ver cuadro 3).
Gráfico $2 \triangleright$ Investigadores extranjeros en el SNI por área, 2013

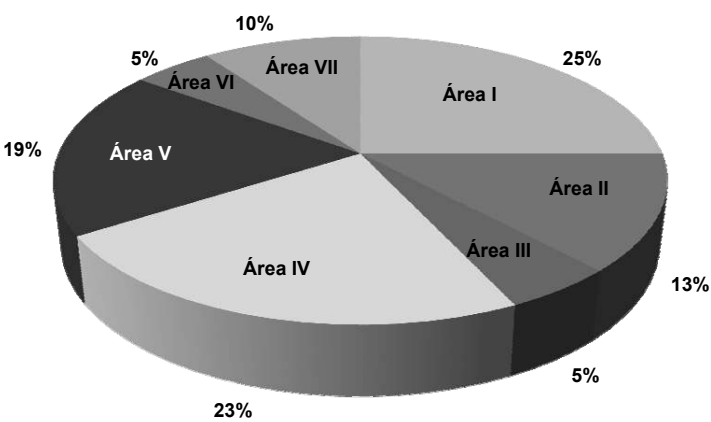

Fuente: CONACYT, SNI Investigadores vigentes, agosto 2013

La muestra fue construida con base en el origen nacional, excluyendo cualquier otro filtro, a partir de una muestra amplia integrada por 124 investigadores extranjeros. En su mayoría, los informantes son miembros del S.N.I y eran titulares de su puesto, aunque algunos tenían un estatuto de post-doc cuando nos

Cuadro $3 \triangleright$ Principales características de la muestra

\begin{tabular}{|c|c|c|c|c|c|}
\hline Registro & Sexo & Año Nacimiento & Lugar obtención grado & $\begin{array}{c}\text { Fecha } \\
\text { titulación }\end{array}$ & $\begin{array}{l}\text { SNI (al momento } \\
\text { entrevista) }\end{array}$ \\
\hline 1 & $\mathrm{~F}$ & 1941 & Nanterre & 1997 & II \\
\hline 2 & $\mathrm{~F}$ & 1976 & Université Bourgogne & 2002 & I \\
\hline 3 & $M$ & & & & $?$ \\
\hline 4 & $\mathrm{~F}$ & 1980 & Milton Keynes, Londres & & $\mathrm{C}$ \\
\hline 5 & $\mathrm{~F}$ & 1937 & UNAM (Física) & & 1 \\
\hline 6 & $\mathrm{~F}$ & 1969 & Aix Marseille 3 & 1997 & II \\
\hline 7 & $\mathrm{~F}$ & 1954 & Sorbonne & & II \\
\hline 8 & $\mathrm{~F}$ & 1964 & EHESS & 1998 & No \\
\hline 9 & $\mathrm{~F}$ & 1967 & Lyon 1 & 1997 & 1 \\
\hline 10 & M & 1941 & & & No \\
\hline 11 & $\mathrm{~F}$ & 1946 & Paris XIII & & II \\
\hline 12 & $\mathrm{~F}$ & & Paris & 1984 & II \\
\hline 13 & M & 1944 & EHESS & 1987 & III \\
\hline 14 & $\mathrm{~F}$ & & 1996, EHESS & & II \\
\hline 15 & M & 1966 & Univ. Paris 1996 & & I \\
\hline 16 & $\mathrm{~F}$ & 1963 & Y. René Descartes 1998 & & No \\
\hline 17 & $M$ & & Paris 3, 2004 & & I \\
\hline 18 & M & & Claude Bernard, Lyon, 1996 & & II \\
\hline 19 & M & 1957 & Rennes 1, 1994 & & II \\
\hline 20 & $M$ & 1979 & COLMEX/Sorbonne 2009 & & I \\
\hline 21 & $M$ & 1952 & Paris VIII & & II \\
\hline
\end{tabular}

Fuente: entrevistas levantadas entre 2011 y 2014 por S. Didou, C. Oviedo y M. Narezo 
concedieron la entrevista. Dos trabajaban por organismos franceses: se encontraban en una situación de movilidad plurianual pero temporal en México, con sueldo pagado por su institución de adscripción.

Todos los informantes tienen injerencia en colectivos de investigación y asociaciones. Varios participan o han participado en programas de apoyo a la investigación, nacionales bilaterales o internacionales; tienen a la par una actividad individual en tanto especialista en un tema de indagación y una colectiva, articulada en distintos niveles. Ocupan posiciones que permiten alcanzar un reconocimiento en los países de procedencia y llegada, se ubican en círculos disciplinarios que avalan su calidad y acceden, puntual o constantemente, según las personas y las áreas disciplinarias: la procuración de recursos externos depende de necesidades, oportunidades y de un cálculo costos-beneficios.

Desde las fases tempranas del proceso de inserción profesional, e incluso antes para los más jóvenes, los individuos trabajan en grupos amplios, en distintos países. Por lo general, desde su época estudiantil, efectúan estancias de movilidad, viajando al exterior para asistir a congresos o realizar prácticas en equipos multilaterales de investigación. El arranque de un proceso de internacionalización es cada vez más precoz. Una de las entrevistadas estudió el doctorado fuera de Francia y, a los 24 años, ya inscrita en doctorado, ganó una beca para insertarse temporalmente en un equipo de 150 doctorantes y postdoctorantes que realizaban trabajo de campo en un país tercero, bajo el liderazgo de un líder mundialmente reconocido. El trabajo en equipo, no solo en un laboratorio específico, que estabiliza los intercambios y norma las relaciones verticales y horizontales entre sus miembros, sino en estructuras abiertas, con un componente intercultural evidente pero inestable, se aprende desde los inicios de la trayectoria científica.

Al llegar a México o en su primera década de estancia en el país, los informantes aplicaron esa táctica de inserción en redes para darse a conocer en el medio científico de recepción e incluso para precisar su línea propia de investigación. Sobresale en general un grato recuerdo de ello:

Cuando la Red de Colegios, en el 2000 dice "queremos nosotros impulsar un proyecto de todos los colegios sobre el tema del agua", entonces vamos a juntar un grupo de investigadores para armar un proyecto para todo el país, en ECOSUR, me mandaron a trabajar esto. Armamos un grupo con gente que tenía muchísima trayectoria en el tema; no estaban el CIESAS o el Instituto Mora en ese momento en la Red pero sí el Colegio de San Luis, el de Michoacán, el Colegio de México, el Mexiquense, el de la Frontera Norte, el de
Sonora, el de Jalisco, etcétera. Para mí, fue un reencuentro con las Ciencias Sociales, fue muy importante [...] me permitió estar en contacto con estos colegas que trabajaban el tema, con los cuales, hasta la fecha de hoy tengo relaciones académicas. (Entrevista n. 6)

La participación en redes de investigación, aun temporales, y las conexiones establecidas con ese pretexto y durante congresos fueron fundamentales en la consecución de un capital de legitimidad, cuando no de un puesto, principalmente entre los más jóvenes.

\section{Llego a México...con mucha suerte. Cuando} se acabó el [segundo] contrato de posdoc, no había nada en Francia. Yo no quería irme de nuevo a otro país, a otro posdoc. Después de 6 años de posdoc, ya estaba harto de esta situación: de no saber a dónde vas, de ¿qué voy a hacer acá? Yo mandé un email a todos mis ex-colegas, conocidos... y me contestó un colega diciendo: "ipor supuesto!, tienes una buena oportunidad para dejar la [disciplina***]... pero sabemos que te gusta mucho". Entonces yo le conteste a él: pues es que yo no tengo nada, yo no quiero irme ya de posdoc. Y un mes después, me mandó un email este investigador que yo había conocido en París, diciendo: mira, tenemos una oportunidad, tenemos que encontrar a alguien para llenar un hueco de una plaza que se liberó... y tenemos que proponer a alguien ahora mismo o se va a perder. Entonces: ¿estas interesado? No es un posdoc, es una plaza fija, por el momento, no tenemos a nadie y tenemos que contestar mañana... Entonces, me tienes que decir, hoy, si estas interesado. Entonces pues sí, yo mandé [mis papeles]... Había venido dos veces a México, por congresos. (Entrevista n. 17)

En contraste, los más viejos suelen narrar una historia de acceso al primer empleo, vinculada con la decisión unilateral de una autoridad universitaria interesada en los saberes específicos de los que eran detentores en periodos en los que la transferencia de conocimientos era menos inmediata y más vinculada a la circulación internacional de los individuos que ahora. En contraste, entre los menores a 45 años, predomina la paciente hilación de una trama de relaciones académicas o sociales y la presentación sucesiva de expedientes de candidatura a proyectos y apoyos. Aunque no tan frecuentemente, sobre todo entre los menores a los 40 años, está un tercer canal de inserción fundamentado en la militancia política:

Entonces mis primeros conectes con universidades públicas en México fueron mediante las 
luchas estudiantiles. Mediante contactos con profesores y estudiantes [de las carreras de] sociología, psicología y humanidades. Después de graduarme, [continuo en Francia] un año más. Obtengo en el mismo año los dos títulos de licenciatura y con relación privilegiada con México, porque fui responsable de una comisión internacional [en un sindicato estudiantil]: entonces organizamos el año siguiente un Encuentro Internacional para la Educación Pública, Gratuita y Laica, o algo así... [En ese $y$ el segundo] que fue en Montreal, yo con mis compañeros invitamos a los mexicanos, los latinoamericanos, otras redes de estudiantes más que investigadores. Pero hoy, hoy, todos o muchos son investigadores. [Entrevista n. 20]

Esas redes de relación, cualquiera que sea su sustento original, desembocan en el acceso a becas de posdoc, reclutamientos temporales e invitaciones en equipos de trabajo, a la par que les provee de un saber hacer trámites, solicitudes y candidaturas. Las experiencias de profesionalización precaria en el extranjero anteceden a la obtención de un puesto académico en México.

A mi ex jefe lo conocí en Francia y me dio la opción de venir, pero la plaza ha sido para mí muy difícil de obtener: he tenido que presentarme a 5 concursos porque tenían perfiles ad hoc, sobre pedido. (Entrevista n. 9)

En efecto, una motivación profesional común entre los jóvenes científicos franceses que entrevistamos fue el escapar a una prolongación excesiva del estatuto de postdoc., compitiendo y obteniendo un puesto en México. En ese sentido, la migración, más que a un interés específico centrado en instalarse en el país de recepción, obedece a factores que obstaculizan el ingreso al mercado científico en el de origen. Remite por ende a una lógica de push más que de pull. En el caso específico, la relativa saturación del mercado científico en Francia, el que el número de doctores y postdoctores en busca de empleo rebase el de puestos disponibles, el acrecentamiento de la competencia internacional para el acceso a las escasas posiciones ofertadas explican que muchos jóvenes empiezan a circular internacionalmente para obtener un empleo estable. Lo corrobora el que en Francia, el éxodo de los recursos humanos altamente calificados haya devenido un objeto de preocupación, como lo indican, desde finales de los 90, la publicación recurrente de reportes expertos respecto del éxodo de los más calificados (Papon, 2004; Ennafaa y Paivandi, 2008). Por el lado de México, el fuerte tejido histórico de relaciones científicas entre ambos países, la formación de un número todavía considerable de investigadores mexicanos en Francia y el desarrollo relativo de programas conjuntos de graduación hacen que el país sea un destino atractivo al brindar posibilidades de reclutamiento, al ofrecer oportunidades de movilidad estudiantil bilateral en el marco de carreras compartidas y permitir una inserción en grupos disciplinares afines (Didou y Renaud, 2015).

Descubrimos, mientras preparábamos solicitudes de becas de doctorado, para venirnos acá... el sistema de co-tutela que aquí tradujeron como co-tutoria y entonces descubro que si se pueden hacer las dos cosas. Así fue... pero, pura tramitología. (Entrevista n. 20)

Esos recorridos tortuosos de profesionalización precaria, cuando están acompañados por publicaciones y actividades de vinculación, son sin embargo útiles para, ulteriormente, contar con la cartera de relaciones que permiten consolidar la trayectoria, en el momento en la que los entrevistados la (re) arrancan en buenas condiciones. Confirman lo señalado por Altbach (2015: 11) cuando advierte que los jóvenes científicos extranjeros encuentran en las instituciones de elite situadas en mercados científicos de prestigio intermedio ${ }^{4}$ oportunidades de empleo atractivas: en México, esas consisten en estabilidad laboral, inserción en grupos constituidos y, vía el CONACYT, fondos de apoyo para asistencia a congresos o proyectos de investigación. Pero esas mismas ventajas se diluyen para investigadores con carreras consolidadas en el extranjero, los cuales son más sensibles a disfuncionamientos del mercado científico nacional: entre esos, destacan la alta dependencia de ingresos totales percibidos por los académicos de los incentivos y sobresueldos por productividad, las condiciones de gestión burocratizada de la investigación, las malas condiciones de jubilación y las insuficiente calidad de los servicios públicos de salud. Los franceses de mediana edad son particularmente sensibles a esas últimas dos cuestiones, al proceder de un país que todavía provee servicios de bienestar de calidad.

Por lo general, los académicos franceses en México no se desligan del todo de su comunidad de origen pero, laboralmente, no siempre Francia es su primera elección como contraparte preferente de cooperación científica. La selección de colegas con los que montar proyectos depende de donde radican los grupos de avanzada en su línea: aunque, por regla general, los equipos referentes son pocos a escala mundial, su dispersión geográfica obliga a sopesar con quiénes es posible trabajar efectivamente, dadas las condiciones de vida académica y los recursos disponibles en México.

Las interacciones con Francia pasan por dos canales: los primeros (ECoS o Cátedras) están manejados localmente, a través de la Embajada de 
Francia, generalmente en cooperación con organismos mexicanos; los segundos se construyen como resultados de una cooperación disciplinaria con pares franceses. Los informantes han valorado los primeros pero han deplorado su índole "semilla" (con pocos recursos, muy etiquetados) y una rendición de cuentas desproporcionada con respecto de los recursos captados. Igualmente, consideran negativo el que las reestructuraciones de la cooperación francesa hayan restringido la autonomía de los organismos locales (incluso de la propia Embajada) para proporcionar, en forma ágil, apoyos ad hoc a la investigación. Eso sin menoscabo de que algunos guarden una grata memoria de su paso por el CEMCA, cuando recién llegados a México e intervengan en eventos o comisiones dictaminadoras como pruebas de una relación científica con el país de origen.

No obstante, cuando los informantes mantienen una relación científica bilateral dinámica y provechosa con Francia, lo hacen directamente con grupos científicos homólogos, utilizando recursos procedentes del CONACYT o bien propios (vía la institución o proyectos externos) para absorber sus costos. Las relaciones se establecen en torno a un proyecto de interés compartido con los colegas. Se van institucionalizando conforme los individuos y grupos demuestran sus capacidades de producción conjunta. Después de cierto tiempo de colaboración de baja intensidad y costo reducido, se ramifican en publicaciones, intercambio de estudiantes, estancias de profesores, co grados o co tutelas, seminarios y congresos e implican eventualmente la firma de convenios.

Por otra parte, los entrevistados manifiestan reiteradamente su interés, independientemente de los anclajes territoriales de las disciplinas, para participar en redes con el fin de comparar situaciones distintas y cotejar perspectivas sobre un mismo objeto. Valoran la interconexión de varias de esas, diferenciadas espacial y disciplinariamente para generar ángulos innovadores de acercamiento a problemas científicos. En ese sentido, lo bilateral es sólo una vertiente de una estrategia de internacionalización multi-situada no sólo con colegas en el país de origen sino con pares, independientemente de su localización geográfica. En cambio, cuando participan en asociaciones y sociedades científicas, esas tienen un carácter de agrupación disciplinaria más que territorial. Los beneficios esperados son de orden personal, al igual que los pagos de las cuotas de afiliación. Consisten en mantenerse al tanto de los avances en su línea de investigación, recibir descuentos para comprar revistas o derechos de inscripción a congresos o enterarse de convocatorias.

\section{Conclusiones}

Los perfiles de formación y desempeño de los investigadores franceses corresponden con los exi- gidos por las instituciones mexicanas de educación superior. La producción inicia en el doctorado con una autonomía incremental en el postdoctorado. Su dominio, en ocasiones, de idiomas adicionales al español, al francés y al inglés les facilita pertenecer a redes, dialogar con colegas extranjeros y publicar en otros países. Los más jóvenes de ellos han dejado atrás el modelo de movilidad bilateral, predominante entre sus antecesores, por uno multi-localizado, asimilando culturas profesionales heterogéneas, socialmente construidas y determinadas por negociaciones y prácticas particulares (Pickering, 1992).

Terminé la licenciatura en Paris. Después la maestría la hice el primer año en Alemania... con un programa que se llama Erasmus...es el programa más conocido de movilidad estudiantil en Europa, es muy famoso. Luego después de la maestría, me fui dos años a Senegal a hacer servicio nacional: era obligatorio en aquella época. [...] Terminando el doctorado en París, ingreso a un posdoc a Brasil y después a otro en Marsella. (Entrevista n. 15)

Independientemente de la edad, la movilidad no caracteriza sólo la etapa inicial de formación sino que influye toda la trayectoria. En parte, explica la adecuación, aparentemente tersa, de las competencias genéricas de los científicos franceses a las exigidas por el medio académico mexicano, altamente internacionalizado en sus parámetros deseables de desempeño (aunque no siempre en sus medios y resultados). Sus experiencias de vida, formación y trabajo en otros países les confieren ventajas en el momento de ocupar plazas vacantes; sin embargo, esas ventajas, muy recordadas y valoradas por los informantes de mayor edad, están diluyéndose paulatinamente para los jóvenes, debido a la creciente competencia para acceder a puestos, en número restringido e inferior a las demandas. Opinan que la internacionalización es un elemento importante en sus procesos de profesionalización, advierten que su grado de valoración depende de cómo las instituciones y los equipos científicos la perciben, en tanto atributo exclusivamente individual o como una característica con repercusiones colectivas benéficas.

Movilizan en consecuencia tácticas de visibilización y socialización relativa de su cartera de relaciones internacionales, mediante una inserción en redes, una participación asociativa y una utilización focalizada de programas bilaterales de cooperación científica. Su propensión a insertarse en esas estructuras, a la vez, les otorga un plus de capital social ante los pares, legitimidad académica en su nicho disciplinario y prestigio en sus instituciones de adscripción, siempre y cuando sean de "beneficio compartido". 
Un análisis de las redes a las que pertenecen los investigadores franceses entrevistados indica su diversidad y su porosidad. Es infrecuente el que un investigador pertenezca a una sola red y que sus redes sean todas "cortadas sobre el mismo patrón". Varios mencionan su adscripción a redes de investigación pero también de discusión e intercambio de estudiantes. En ese sentido, la cuestión de qué objetos o productos se mueven en el interior de las redes (que tanto interpeló a los especialistas hace unos quince años - Casas, 2001) encuentra respuestas variadas. Mediante las redes, los investigadores franceses tratan de conseguir, según el modelo canónico, recursos y medios para avanzar en el conocimiento de un tópico y abrir perspectivas innovadoras sobre una línea de indagación mediante una confrontación de perspectivas particulares. Redistribuyen por ese medio parte de las ventajas obtenidas a los actores que conforman sus circuitos principales y naturales de apoyo profesional - estudiantes, asistentes, investigadores menos consolidados. Discuten en ellas preocupaciones teóricas, avances, instrumentos, normas o incluso controversias es decir abren espacios de aprendizaje compartido y mutuo, tácito y explícito.

A su vez, en más redes intervenga un líder de investigación y más dispuesto esté en interconectarlas, mayor es la probabilidad de que el (los) colectivo(s) institucional(es) o disciplinario(s) al(os) que pertenece reciban beneficios, directos o indirectos. En las redes, las pertenencias individuales tienden a tener repercusiones positivas sobre el grupo fungiendo, independientemente de las disciplinas, como "estructuras relacionales que garantizan una capacidad de producir y de innovar". En su interior se enmarcan interdependencias racionalizadas entre asociados "rivales" o jerarquizados, en el entendido de que en los "análisis institucionales y organizacionales clásicos, no es extraño encontrar teorías sobre la importancia de las redes en la capacidad individual de innovar", (Latour y Woolgar, 1979) pero también en la capacidad colectiva de innovar, facilitando procesos orientados a "co-orientarse y aprender colectivamente" (Lazega, 2004: 1-2).

En contraste con las redes, orientadas a garantizar beneficios colectivos e individuales mediante la socialización de prácticas, conocimientos y recursos, las elecciones "asociativas" están al parecer articuladas al revés: predomina la idea del beneficio propio e individualizado mediante una participación personal. Por lo general, los entrevistados justifican las pertenencias asociativas y el uso de programas de cooperación bilateral en términos de las ventajas que proporcionan al individuo más que en términos de compromisos socio-profesionales amplios.

Finalmente, dado que los entrevistados son de muy diversa edad, se han insertado en un mercado científico que funciona de forma distinta, sobre todo tomando en consideración las políticas de reestructuración del cual ha sido objeto en las tres décadas pasadas por parte del gobierno. Igualmente, sus procesos de socialización científica, previos a la migración, han sido diferentes en Francia. Mientras los de mayor edad fueron invitados a México por ser "portadores" específicos de saberes y de teorías, los más jóvenes acceden a un puesto logrando ganar una competición entre varios candidatos pero sin estar específicamente distinguidos como "la" persona a contratar. Ese cambio en los procesos de inserción profesional a su vez incide en las ventajas relativas otorgadas a los académicos extranjeros pero también en sus oportunidades de transferencia de saberes, en una lógica de dominación intelectual Norte-Sur. Eso es un punto central para el estudio de los fenómenos de neo-colonialismo que habría que tratar con detenimiento en investigaciones ulteriores.

\section{Notas}

1 Con el soporte de la Red sobre Internacionalización y Movilidades Académicas y Científicas (RIMAC), Programa de Redes Temáticas del CONACYT n 260402.

2 http://www.ctifranciamexico.com/index.php/component/ sobipro/151 ? Itemid $=359$

3 http://www.ctifranciamexico.com/index.php/investigacion-e-innovacion/la-cooperacion-franco-mexicana

4 Por mercado científico de prestigio intermedio, entendemos un sistema científico que ha mejorado sus niveles de desempeño, publicaciones internacionales, revistas acreditadas y cuenta con dispositivos complejos de aseguramiento de calidad, carreras académicas formalizadas, centros especializados de investigación, algunos de los cuales podrían ser clasificados como research universities, grupos de investigación reconocidos internacionalmente en ciertas áreas y una oferta de formación de posgrado en los tres niveles de especialización, maestría y doctorado. Sin embargo, sus niveles de producción académica en revistas mainstream, de obtención de patentes y de participación en rankings son todavía relativamente bajos.

\section{Referencias bibliográficas}

AGULHON, C. (2015), "Circulación de los individuos-circulación de los saberes Francia-México, de una generación a otra", en Didou Aupetit, S. \& P. Renaud (Coord.), op-cit, pp. 71-94. Disponible en: http://www.iesalc.unesco.org.ve/index. php?option=com_fabrik\&view=details\&formid = 2\&rowid=177\&lang=es

AINSWORTH, S. et al. (2015), "Mapeo de la colaboración en ciencia y tecnología entre México y Francia a través de un análisis de co-publicaciones 1984-2010", en Kleiche-Dray, M. \& D. Villavicencio (Coord.), op-cit, pp. 49-74. Disponible en: http://biblioteca. clacso.edu.ar/clacso/posgrados/20141028014136/ cooperacion.pdf

ALBUQUERQUE, G. (2015), "Los cientistas sociales latinoamericanos en la Revue Tiers Monde de Paris (1960-2010). Un caso de circulación internacional 
de conocimiento", en Didou Aupetit, S. \& P. Renaud, op-cit, pp. 255-268.

ALTBACH, P. (2015), "Building an Academic Career: A Twenty-First-Century Challenge", en Yudkevich, M; Altbach, P. Y L. Rumbley. (Eds.), Young Faculty in the Twenty-First Century: International Perspectives, Estados Unidos, Suny Press, pp. 5-18. Disponible en: http://www.sunypress.edu/ pdf/63028.pdf

APPELT, S. et al. (2015), "Which factors influence the international mobility of research scientists?" en OECD Science, Technology and Industry Working Papers, 2015/02, Paris, OECD Publishing. Disponible en: http://www.oecd-ilibrary.org/ docserver/download/5js1tmrr2233.pdf?expires= 1439486688\&id $=$ id\&accname $=$ guest\&checksum $=$ 36BE183A8A981A672E8ECAD02515236E

BATAILLON, F. \& F. GIRAUD (1986), IFAL, 1945-1984. Histoire de I'Institut Français d'Amérique Latine, México, IFAL.

BERTRAND, M. \& C. PAILLER (2013), "Regards sur 50 ans de latino-américanisme" en Caravelle, No. 100, Junio, 318 p. Disponible en: http://www.decitre.fr/ revues/caravelle-n-100-juin-2013-regards-sur-50ans-de-latino-americanisme-9782810702633.html

CASAS, R. (coord.) (2001), La formación de redes de conocimiento: una perspectiva regional desde México, España, Anthropos-UNAM. Disponible en: https://books.google.com.mx/books?id= JAQPWjKGD-cC\&printsec $=$ frontcover $\& d q=$ re des+de+conocimiento\&hl=es\&sa =X\&ve$\mathrm{d}=0$ CBwQ6AEwAGoVChMIjvnr9qSTxwIVjwaSCh3C $w k P \# v=$ onepage $\& q=$ redes $\% 20$ de $\% 20$ conocimiento\& $\mathrm{f}=$ false

CASTAÑOS, H. (2009), "Migración internacional de y hacia México", en Leite, P. y S.En Giorguli (coord.), El estado de la migración. Las políticas públicas ante los retos de la migración mexicana a Estados Unidos, México, Consejo Nacional de Población, pp. 345-368.

CASTAÑOS, H. et al. (2004), La migración de talentos en México, México, D.F. IIEC-UNAM- Porrúa. Disponible en: http://ru.iiec.unam.mx/2053/1/ LaMigracionDeLosTalentos.pdf

DIDOU, S. \& J. P. Durand Villalobos (2013), "Extranjeros en el campo científico mexicano: primeras aproximaciones", en REDIE, Revista Electrónica de Investigación Educativa, Vol. 15, No. 3, pp. 68-84. Disponible en: http://redie.uabc.mx/index. $\mathrm{php} / \mathrm{redie} /$ article/view/557

DIDOU, S. (2014a), Cooperación franco-mexicana en educación superior y ciencia: irepunte o reestructuración? [en línea]. OBSMAC, Opiniones de Expertos [consultado en agosto de 2015]. Disponible en: http://unesco.org.ve/index.php?option $=$ com $_{-}$ content\&view $=$ article\&id=3519: cooperacion-franco-mexicana-en-educacion-superior-y-ciencia-repunte-o-reestructuracion\&catid $=201$ : experiencias-y-programas\&Itemid $=770 \&$ lang $=e s$

DIDOU, S. (2014b), "Investigadores franceses en México: inserción profesional y redes de trabajo", en KleicheDray, M. \& D. Villavicencio, op. cit, pp. 139-158.
DIDOU. S. \& P. Renaud (2015), Circulación Internacional de los Conocimientos: Miradas Cruzadas sobre la Dinámica Norte-Sur, Lima, UNESCO-IESALC. Disponible en: http://www.iesalc.unesco.org.ve/ index.php?option=com_fabrik\&view=details\&for$\mathrm{mid}=2$ \& rowid $=177$ \&lang $=$ es

DURAND VILLALOBOS, J. P. (2013), Científicos extranjeros en la Universidad de Sonora: contribuciones e impacto en las comunidades disciplinares locales. Reseña de tesis, Venezuela, OBSMAC-IESALC. Disponible en: http://www.unesco.org.ve/index. $\mathrm{php} / \mathrm{pt} / \mathrm{component/fabrik/details/9/14}$

ENNAFAA, R. y S. Paivandi (2008), Les étudiants étrangers en France. Enquête sur les projets, les parcours et les conditions de vie, Paris, La Documentation française. Disponible en: http://www.ladocumentationfrancaise.fr/catalogue/9782 110068613/

ESPENSHADE, T.; M. Usdansky \& C. Chung (2001), "Employment and earnings of foreign-born scientists and engineers" en Population Research and Policy Review, Vol. 20, No. 1-2, pp. 81-105. Disponible en: http://link.springer.com/article/10. 1023\%2FA\%3A1010660808072?LI=true\#page-1

FRANZONI, C.; G. Scellato \& P. Stephan (2012), "Foreign Born Scientists: Mobility Patterns for Sixteen Countries", en NBER Working Paper, No. 18067. Disponible en: http://www.nber.org/papers/ w18067.pdf

FRANZONI, C.; G. Scellato \& P. Stephan (2013), "The Mover's Advantage: The Superior Performance of Migrant Scientists", en Economic Letters, Vol. 122 , No. 1, pp. 89-93. Disponible en http://isites. harvard.edu/fs/docs/icb.topic1258829.files/2-2814_STEPHAN-Paula_3_Movers-Advantage-Migrant-Scientists_1-10-14.pdf

GALAZ FONTES, J. et al. (2009), "The academic profession in Mexico: Changes, continuities and challenges derived from a comparison of two national surveys 15 years apart", en RIHE International Seminar Reports, N. 13, pp. 193-212. Disponible en http://www.rdisa.org.mx/documentos/ Productos $\% 20$ RPAM/The $\% 20$ Academic $\% 20$ Profession $\% 20$ in $\% 20$ Mexico-\%20Changes $\% 20$ and\%20Continuities\%202009.pdf

GERARD, E. (2013), "Dynamiques de formation internationale et production d'élites académiques au Mexique", en Revue d'anthropologie des connaissances, Vol. 7, No. 1, pp. 317-344. Disponible en: http://www.cairn.info/article. php?ID_ARTICLE $=$ RAC_018_0317

GREDIAGA, R. \& E. Maldonado (2014), "Relación científica México-Francia. Influencia de los principales polos internacionales en el desarrollo y evolución de la Biotecnología en México", en Kleiche-Dray, M. \& D. Villavicencio, op. cit, pp. 177-212.

HEBRARD, V. (2012), "François Chevalier (1914-2012)", en Bulletin de l'Institut français d'études andines, Vol. 41, No. 2. Disponible en: http://bifea.revues. org/685

HEYMANN, C. (2015), Construcción y evolución de los conocimientos sobre México en la revista francesa 
Les Langues Néo-Latines. Disponible en http:// www.rimac. $\mathrm{mx}$

HUERTA, M. (2006), "Un mediateur efficace pour la cooperation scientifique française : le groupement des universites et des grandes écoles de France pour les relations avec I'Amérique Latine.", en Cuentro de Latinoamericanistas Españoles (12. 2006. Santander): Viejas y nuevas alianzas entre América Latina y España. CEEIB, pp. 792-803. Disponible en: https://halshs.archives-ouvertes. $\mathrm{fr} /$ halshs-00103840/document

KLEICHE-DRAY, M. \& D. Villavicencio (coords.) (2015), Cooperación, colaboración científica y movilidad internacional en América Latina, Buenos Aires, CLACSO. Disponible en: http://biblioteca.clacso. edu.ar/clacso/posgrados/20141028014136/ cooperacion.pdf

LATOUR, B. y S. Woolgar (1979), Laboratory Life. The Construction of Scientific Facts, Princeton, Princeton University Press. Disponible en: http://home. ku.edu.tr/ mbaker/CSHS503/LatourLabLif.pdf

LAZEGA, E. et al. (2004), "Discipline scientifique et discipline sociale: Réseaux de conseil, apprentissage collectif et innovation dans la recherche française sur le cancer (1997-1999)", en Recherches Sociologiques, No. 2. Disponible en: http://elazega.fr/ media/pdf/art/DSDS-2004.pdf

LEYDESDORFF, L. et al. (2013), International Collaboration in Science: The Global Map and the Networt, Cornel University Library. Disponible en http:// arxiv.org/ftp/arxiv/papers/1301/1301.0801.pdf

LIN, Z., R. Pearce \& W. Wang (2009), "Imported talents: demographic characteristics, achievement and job satisfaction of foreign born full time faculty in four-year American colleges", en Higher Education, Vol. 57, No. 6, pp. 703-721. Disponible en: http://link.springer.com/article/10.1007/s10734008-9171-z\#page-1

MEYER, J. (s.f.), "Los franceses en México durante el siglo XIX", en Revista Relaciones, 2 (1), pp. 5-54. Disponible en: http://www.revistarelaciones.com/ files/revistas/002/JeanMeyer.pdf

OVIEDO, M. (2015), "Redes de trabajo de investigadores nacidos fuera de México. Algunas reflexiones", Productos académicos. Red sobre Internacionalización y Movilidades Académicas y Científicas-RIMAC. Disponible en: http://www.rimac.mx/redes-de-trabajo-de-investigadores-nacidos-fuera-de-mexico-algunas-reflexiones-1/

PAPON, P. (2004), "Fuite des cervaux, "Brain Drain" et "Brain Gain", Colloques de la Fondation Res Publica, Paris, Fondation Res Publica. Disponible en: http://www.fondation-res-publica.org/Fuitedes-cerveaux-Brain-Drain-et-Brain-Gain_a30.html

PÉREZ SILLER, J. \& C. CRAMAUSSEL (2004), México Francia: memoria de una sensibilidad común, siglos $X I X-X X$, Volumén 2, México, El Colegio de Michoacán A.C. Disponible en: https://books.google.com.mx/books?id=ZtRZI$z Q \mid r Q g C \& p g=P A 136 \& l p g=P A 136 \& d q=$ influen- cia +historia +francesa+en + mexico\&sour$\mathrm{ce}=\mathrm{bl} \&$ ots $=$ WvK5rQA6z7\&sig $=I C-K V e h-$ HPae9Irjl6p6TybW3v6o\&hl=es-419\&sa =X\&ve$\mathrm{d}=0$ CEoQ6AEwB2oVChMIgraX3JSQXwIVR6 WICh22vAAA\# $v=$ onepage\& $q=$ influencia\%20historia\%20francesa\%20en\%20mexico\&f=false

PICKERING, A. (1992), Science as Practice and Culture, USA, The University of Chicago Press. Disponible en https://books.google.com.mx/ books? $\mathrm{hl}=\mathrm{es} \& \mathrm{I}=\& \mathrm{id}=\mathrm{U} \_\mathrm{mL} 2 \mathrm{pPGY7YC} \& \mathrm{o}=\mathrm{fnd} \&$ $p g=P R 7 \& d q=$ Scientific + culture\&ots $=f R-X H 2 h-$ vTC\&sig=X_p3MUcSZnDzZQ_6solUdYerikU\#v=0 nepage \&q=Scientific\%20culture\&f=false

ROLLAND, D. (2002), "El exilio francés en México durante la segunda Guerra Mundial", en Yankelevich, P. (Coord), México, país refugio: la experiencia de los exilios en el siglo XX, México, Instituto Nacional de Antropología e Historia, pp. 101-118.

SABHARWAL, M. (2008), Job Satisfaction of Foreign-born Faculty in Science and Engineering by Citizenship Status, Eisenhower Parkway, ProQuest. Disponible en: http://books.google.com.mx/books?id=kGHS $3 \mathrm{IQg} 1 \mathrm{GgC} \& \mathrm{pg}=$ PA150\&lpg=PA150\&dq=foreign $+b$ orn + scientists \&source $=$ bl\&ots $=o N Y t 34 S H f g \& s i g=$ MabZwnJRH3D6z_IGXz2-SgqiM-4\&hl=es-419\&sa= $X \&$ ei $=$ dCuiU6T3BY6KqAaquICoCg\&ved =0CF4Q6 AEwDTgU \# $v=$ onepage $\& q=$ foreign $\% 20$ born $\% 20$ scientists\&f=false

SÁNCHEZ, G. (2012), "In memoriam, Francois Chevalier (1914-2012) Nota necrológica", en Revista de Estudios Históricos Tzintzun, No. 56, pp. 249-253. Disponible en: http://www. scielo.org.mx/scielo.php?script $=$ sci_arttext\&pi $\mathrm{d}=\mathrm{S} 0188-28722012000200011 \& \mathrm{lng}=$ pt\&nrm $=$ iso

STAMM, C. (2009), "Un geógrafo francés en América Latina. Cuarenta años de recuerdos y reflexiones sobre México", en Investigaciones Geográficas, No. 69, pp. 140-162. Disponible en: http://www. scielo.org.mx/scielo.php?script=sci_arttext\&pi $d=$ S0188-46112009000200014

TEICHLER, U. (2015), "Academic Mobility and Migration: What We Know and What We Do Not Know", European Review 05/2015; 23 (S1), S6-S37. Disponible en http://www.researchgate.net/ publication/277615483_Academic_Mobility_and_ Migration_What_We_Know_and_What_We_Do_ Not_Know

VILLAVICENCIO, D. (2014), "Algunas reflexiones sobre la cooperación científica y universitaria entre Francia y México de los últimos años", en Kleiche, M. \& D. Villavicencio, op. cit., pp. 95-117.

WAGNER, A. C. \& D. García Garza (2015), "¿Estudiar en las escuelas de negocios y de poder en Francia? Los estudiantes mexicanos en administración y ciencia política en las Grandes Écoles francesas", en Didou. S. \& P. Renaud, op. cit, pp. 95-120.

WALTER, J. \& D. Scheltzer (2014), "Pioneros, institucionalización y profesionalización de los estudios del trabajo en América Latina", en Kleiche, M. \& D. Villavicencio op. cit, pp. 213-234. 
Recebido a 12/10/2015. Aceite para publicação a 03/12/2015.

Sylvie Didou Aupetit (didou@cinvestav.mx). Centro de Investigación y Estudios Avanzados, Departamento de Investigaciones Educativas (Cinvestav-DIE). Calzada de los Tenorios, 235, Col. Granjas Coapa, México DF, 04330 México.

María Cecilia Oviedo Mendiola (moviedo@cinvestav.mx). Centro de Investigación y Estudios Avanzados, Departamento de Investigaciones Educativas (Cinvestav-DIE). Calzada de los Tenorios, 235, Col. Granjas Coapa, México DF, 04330 México. 